\title{
Nanoparticles and Cells: An Interdisciplinary Approach
}

\author{
Alke Petri-Fink ${ }^{\star a b}$ and Barbara Rothen-Rutishauser ${ }^{\star a}$
}

\begin{abstract}
In this article we present an overview of some of our research in the field of nanoscience. By combining two different scientific backgrounds (chemistry and biology), we investigate nanoparticle-cell interactions from different angles. This requires an interdisciplinary approach involving material synthesis and characterization, cell biology (biochemistry) and microscopy. In particular, we describe the synthesis and magnetic properties of superparamagnetic iron oxide nanoparticles as well as their behavior in cell culture, evaluate different visualization and detection methods, and investigate the interaction of such magnetic particles with immune cells.
\end{abstract}

Keywords: Dendritic cells · Microscopy $\cdot$ Nanoparticles $\cdot$ Particle-cell interaction $\cdot$ SPIONs
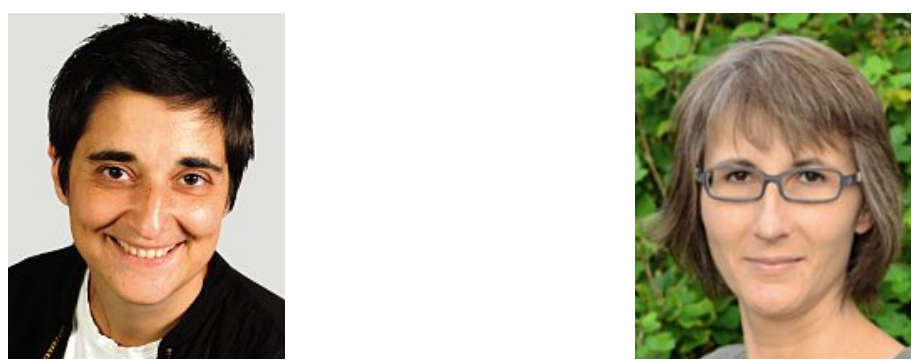

Prof. Dr. Alke Petri-Fink studied chemistry at the University of Ulm, Germany, where she also obtained her $\mathrm{PhD}$ in inorganic chemistry in 1999. After a postdoctoral visit at the Engineering Research Center for Particle Science and Technology, University of Florida, she joined the Institute of Materials Science at the École Polytechnique Fédéral Lausanne (EPFL) in 2000. There she established nano-materials as a new research field, initiated various national and international projects and fostered contacts to many industrial partners. In 2009, she established her own research group as a Swiss National Science Foundation professor in the Chemistry Department at the University of Fribourg. Since July 2011 she is full professor and the new chair of BioNanomaterials at the Adolphe Merkle Institute, University of Fribourg, Switzerland. She shares this position equally with Prof. Barbara RothenRutishauser.

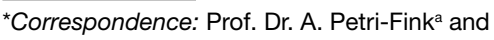
Prof. Dr. B. Rothen Rutishauser

adolphe Merkle Institute

University of Fribourg

Rte de l'Ancienne Papeterie

P.O. Box 209

$\mathrm{CH}-1723$ Marly 1

Tel.: +41263009254

Fax: +41263009624

E-mail: alke.fink@unifr.ch, barbara.rothen@unifr.ch

${ }^{\mathrm{b}}$ Chemistry Department

University of Fribourg

Chemin du Musée 9

$\mathrm{CH}-1700$ Fribourg
Prof. Dr. Barbara Rothen-Rutishauser has received her Ph.D. in 1996 in cell biology at the Swiss Federal Institute of Technology (ETH) in Zurich. From 1996 to 2000 she held a post-doctoral position in Biopharmacy at the Institute of Pharmaceutical Sciences at the ETH where she developed and characterized cell culture models for drug transport studies. In 2000 she joined Prof. Peter Gehr's research group at the Institute of Anatomy, University of Bern, Switzerland. Since 2011 she is an independent group leader at the Respiratory Medicine, Department of Clinical Research and Bern University Hospital, Switzerland, and since $1^{\text {st }}$ July, 2011 she is the new chair in BioNanomaterials at the Adolphe Merkle Institute, University of Fribourg, Switzerland, the position is shared equally with Prof. Alke Fink.

The research of our group focuses around all concepts of nanoscience. By combining various aspects of this scientific discipline, the group of Prof. Alke Fink and Prof. Barbara Rothen-Rutishauser is in a unique position to study and develop bio(nano)materials from their initial synthesis and characterization, to thorough understanding how they may interact with biological systems. In our newly formed research group, the chair position is shared equally between Prof. Alke Fink and Prof. Barbara Rothen-Rutishauser.
This situation is a fresh, novel and exciting perspective upon scientific research in an academic setting, enabling the unification of two different scientific backgrounds together in order to make a truly strong interdisciplinary research group. Prof. Alke Fink leads the material science aspect of the group, whereas Prof. Barbara RothenRutishauser is responsible for all biological and/or toxicological parts. In general we are interested in novel multifunctional and/or hybrid nanomaterials, in particular nanoparticles, for mostly biological and/or medical applications.

The specialty of the group is the synthesis of tailor-made nanoparticles and nano building blocks and their in-depth characterization and integration in different matrices. Research is focused on the development of reactors for reliable, fast, and efficient surface derivatization of nanoparticles, on the interaction of nanoparticles with cells and their colloidal behavior in biological and physiological environments, and on the development of nanoparticle suspensions with tunable optical properties. In addition, many of our current projects investigate nanoparticle-cell interactions in relation to the lung. We are intending to relay this knowledge to promote the safe use of engineered nanoparticles by considering possible health risks of such new bio(nano)materials. Here, our applied projects foci are (i) optimization of cell culture models and aerosol deposition, (ii) visualization and detection of nanoparticles at high resolution, (iii) risk assessment of inhaled bio(nano)materials, and (iv) delivery of new drug systems to the lung.

The rapid expansion of nanotechnology has resulted in the production of an impressive variety of nanomaterials, in particular nanoparticles. Due to their small size and therefore novel physical proper- 
ties and their high surface-to-volume ratio, nanoparticles (NPs) offer novel approaches in a variety of applications. ${ }^{[1]}$ The products launched show clear differentiation across sectors. In general, materials applications are launching first, followed by electronics/IT applications, whereas biomedical applications have a much longer time to market. ${ }^{[2]}$ In contrast to the extensive literature available on the synthesis, the physicochemical properties, as well as on the potential novel applications of innovative and smart nanomaterials, information remains fragmented. The immense potential of nano-sized particles for diagnostic and therapeutic applications requires a thorough understanding on how these NPs interact with living matter such as cellular systems, and their detection and localization within cells is of central importance. Once intracellular NPs are identified, their distribution in different cellular compartments, such as endosomes, lysosomes, mitochondria, the nucleus or cytosol, may also provide some indications as to a potential diagnostic or drug delivery system.

Possible candidates for a variety of biomedical applications are magnetic oxides. Superparamagnetic iron oxide nanoparticles (SPION) are (potentially) used for a variety of biomedical applications such as magnetic resonance imaging (MRI) for contrast enhancement, drug delivery, stem cell tracking, or magnetic separation technologies and ultrasensitive diagnostic assays (Fig. 1). [3]

Some applications are already well estababout fundamental biological interactions

lished (e.g. magnetic separation, MRI), whereas others are still lagging behind. In addition, these particles offer many advantages compared to other tools such as their outstanding magnetic properties, their controllable sizes ranging from about 10 to several hundred nanometers and their custom surface derivatization. For many applications (e.g. MRI, drug delivery), particle sizes below $20 \mathrm{~nm}$ are envisaged to fully exploit those magnetic properties. Then, each nanoparticle shows superparamagnetic behavior above the socalled blocking temperature. In contrast to ferromagnetic or paramagnetic materials, superparamagnetic materials have a high saturation magnetization and negligible coercivity and remanence, as shown in Fig. 2. This superparamagnetic behavior is characterized by a typical relaxation time $\tau$, i.e. the time to return to zero magnetization after an external magnetic field is switched off:

$$
\tau=\tau_{0} \exp \left(\mathrm{KV} / \mathrm{k}_{\mathrm{b}} \mathrm{T}\right)
$$

where $\tau_{0}$ is the characteristic time, $\mathrm{K}$ the anisotropy energy, $\mathrm{V}$ the volume of the particle, $\mathrm{k}_{\mathrm{b}}$ the Boltzmann constant, and $T$ the temperature. Néel losses occur with decreasing particle size, when the thermal activation of reorientation processes leads (in dependence on temperature and measurement frequency) to superparamagnetic behavior of the particle ensemble. ${ }^{[4]}$ Superparamagnetic nanoparticles also allow for heating (of tissue for example)

\begin{tabular}{|c|c|c|c|c|c|}
\hline Purification & $\begin{array}{c}\text { Magnetic } \\
\text { enrichment }\end{array}$ & Hyperthermia & $\begin{array}{c}\text { Drug } \\
\text { delivery }\end{array}$ & Imaging & Cell tracking \\
\hline Separation & Therapy & Diagnosis \\
\hline
\end{tabular}

Multifunctional magnetic nanoparticles

Fig. 1. Typical applications of superparamagnetic iron oxide nanoparticles.

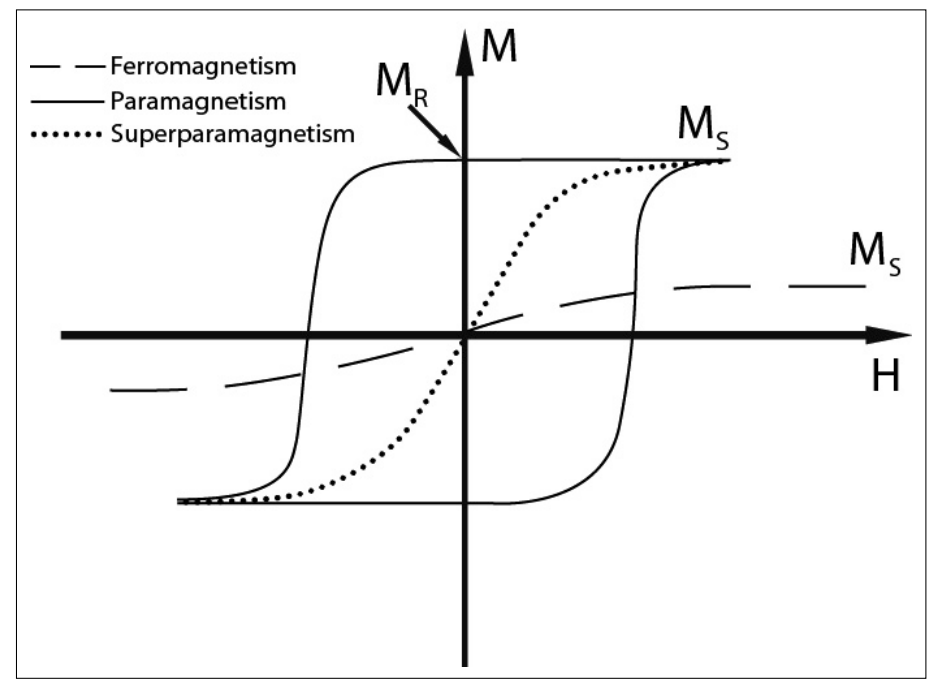

through magnetic relaxation processes. ${ }^{[5,6]}$ In several studies we have investigated the use of in situ implant formation as a form of minimally invasive treatment of cancer lesions by magnetically induced local hyperthermia.[7] Therefore, we have developed injectable formulations (based on alginate or on a poly(ethylene-co-vinyl alcohol) (EVAL)) that form gels entrapping magnetic particles into a tumor. The magnetic particles were embedded in silica microparticles to (i) limit inter-particle coupling through SPION-dipole interactions, (ii) favor Néel relaxation and (iii) allow for high syringeability of the liquid formulations. ${ }^{[8]}$ With this confinement in the solid tumor, we could demonstrate the efficiency of heating in an animal model and the potential for local hyperthermia associated with such a formulation. [9]

In our group, we have established expertise in the field of controlled synthesis, of inorganic nanoparticles, such as superparamagnetic iron oxide NPs, silica, gold, titania, or silver NPs. We differentiate in general between (i) bare, (ii) coated, (iii) core shell and (iv) encapsulated NPs, as illustrated in Fig. 3. Concerning SPIONs, their controlled synthesis represents one of the most important challenges that will determine particle size and shape, particle size distribution, surface chemistry and also particle magnetic properties. Since the blocking temperature depends on the particle size, a broad size distribution will result in a non-ideal magnetic behavior for many applications. ${ }^{[10]}$ Also, crystallinity and (im)purity, and with that magnetic properties, depend on the formation mechanism. Superparamagnetic iron oxide nanoparticles have been synthesized by a large variety of methods, such as coprecipitation, thermal decomposition and/ or reduction, micelle and hydrothermal synthesis, or pyrolysis, each of which has its own advantages and disadvantages, respectively. ${ }^{[10]}$ The co-precipitation synthesis for example - a popular method among chemists - is the most straightforward approach to synthesize nanosized iron oxide particles from aqueous solution (Fig. 4). ${ }^{[11]}$ However, size, shape, and composition of the resulting particles strongly depend on the reaction conditions, in particular the used reactants, $\mathrm{pH}$, and ionic strength of the media. Almost monodisperse magnetic nanoparticles (Fig. 5) can essentially be synthesized by high temperature thermal decomposition of organometallic precursors in the presence of stabilizers. ${ }^{[12]}$ Although the particle size distribution can be much better controlled following these organic decomposition pathways, the final particles are dispersed in organic solvents and need to be transferred to an aqueous environment for any biological and/or medical applications. 


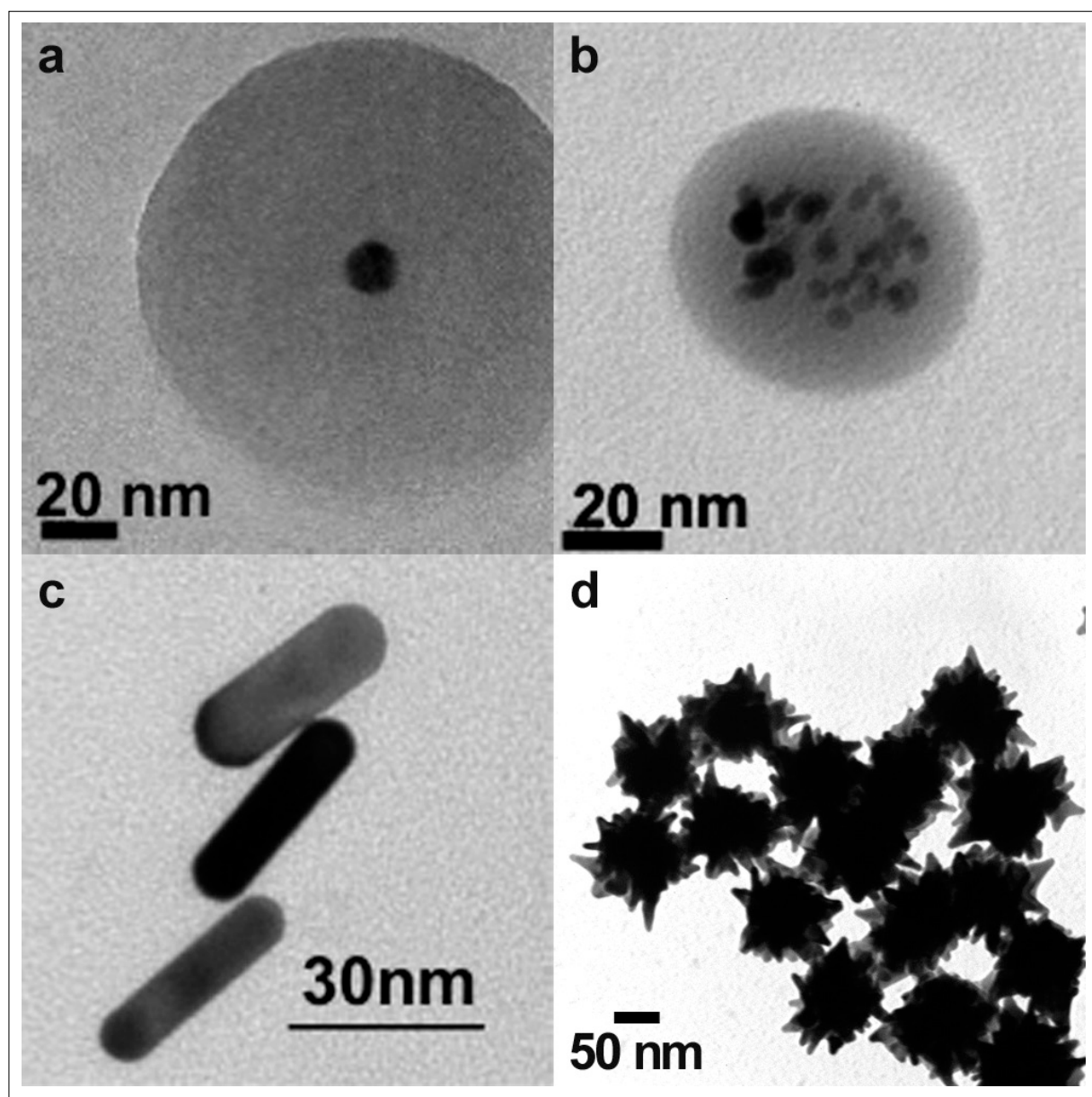

Fig. 3. Transmission electron micrographs of a selection of nanoscale materials (a: silica coated gold NPs, b: SPIONs embedded in a chitosan matrix, c: gold nanorods, d: gold stars).

As mentioned before, the use of inorganic materials, such as silica, is widely reported in the literature to coat magnetic nanoparticles. In order to prepare magnetically adjustable functional hybrid silica magnetic nanoparticles, we have recently condensed a mixture of aminopropyl-triethoxysilane (APTES) and tetraethoxysilane (TEOS) on the surface of previously synthesized superparamagnetic iron oxide nanoparticles. The beads (Fig.

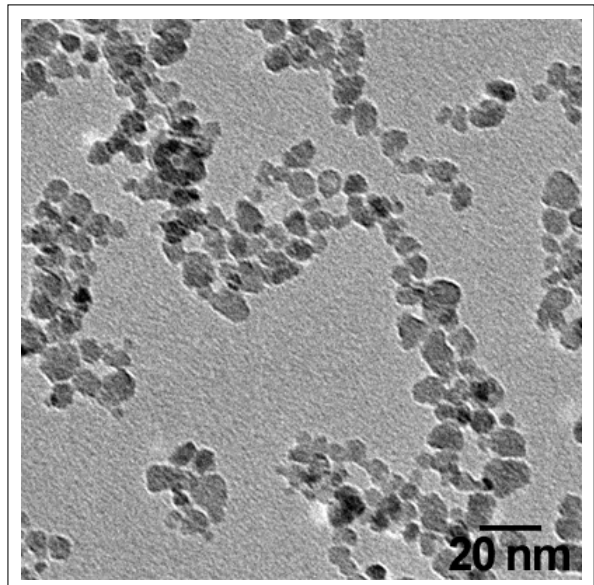

Fig. 4. Transmission electron micrograph of SPIONs (aqueous synthesis).
6) were prepared to allow tuning (i) the size, (ii) the number of accessible amine groups (which are important for any subsequent chemical derivatization) and (iii) the magnetic properties of the final SPION containing beads. ${ }^{[13]}$

In general, significant improvements in the synthesis of (magnetic) nanoparticles have provided the community with protocols to reproducibly synthesize sizecontrolled (magnetic) nanoparticles. Apart

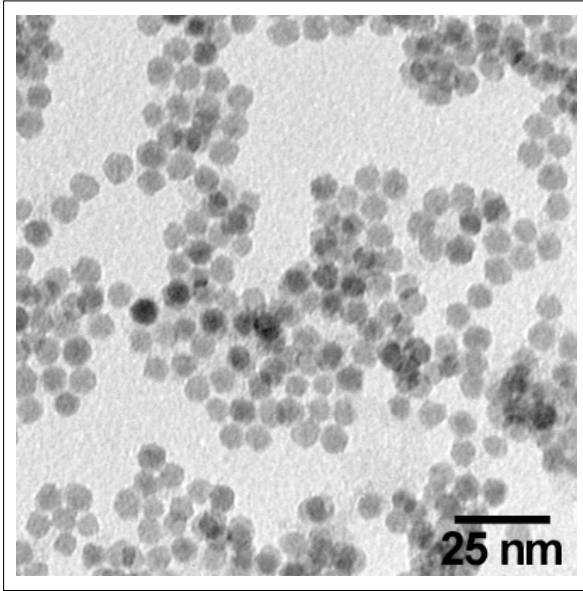

Fig. 5. Transmission electron micrograph of SPIONs (organic synthesis). from the synthesis of the core (magnetic) nanoparticle, the surface becomes more and more important especially for ultrasmall nanoparticles where a large percentage of all the atoms of the particles are surface atoms. Surface effects can, for example, lead to a decrease of the magnetization of nanoparticles as compared to the bulk value. This effect was explained by different mechanisms, such as the existence of a magnetically dead layer on the particle's surface. ${ }^{[14]}$ In addition, the surface plays a crucial role (i) in colloidal stability, (ii) for sophisticated (multifunctional) surface derivatizations, and (iii) for particle-cell interactions. Colloidal stability is a key requirement for almost any application of magnetic nanoparticles. On the one hand, a large variety of surfactants such as synthetic polymers, proteins, or carbohydrates are used for surface coatings, on the other hand inorganic materials such as oxides (e.g. silica) or metals (e.g. gold) are deposited as thin layers on the core magnetic nanoparticles. For many (bio)medical applications, highly complex multifunctional nanoparticles are required. For example, one can imagine a nanoparticle that can target a specific tissue or cell type and at the same time deliver a contrast agent that enables noninvasive imaging and a therapeutic payload to the target. ${ }^{[15]}$

However, surface functionalization of nanoparticles in general still remains a difficult task since colloidal stability during surface derivatization is critical.

In the past, we have developed a fixed bed reactor with a quadrupole repulsive arrangement of permanent magnets to allow magnetic immobilization of the particles in order to (i) perform the derivatization step(s) on the immobilized particles, [16] (ii) facilitate purification, and (iii) easily exchange solvents (Fig. 7). In this magnetic fixed bed reactor the immobilization of the magnetic particles is based on the attractive force exerted on the magnetized

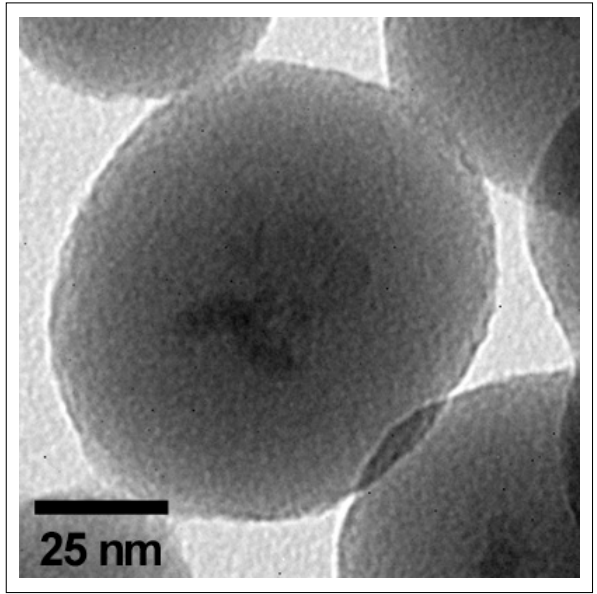

Fig. 6. Transmission electron micrograph of SPIONs embedded in aminopropyl-triethoxysilane. 


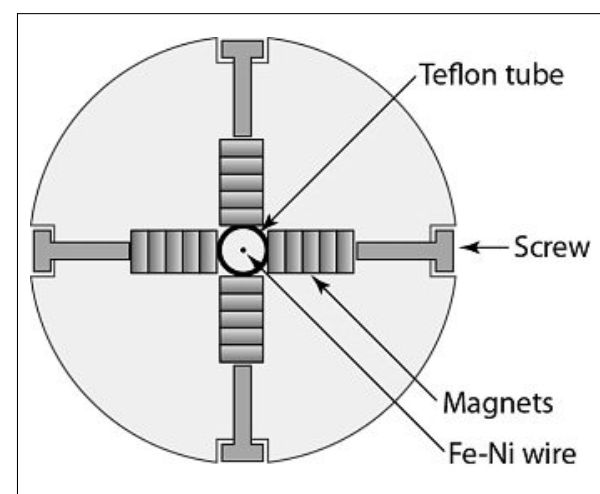

Fig. 7. Scheme of the magnetic reactor.

particles by a magnetic field gradient. In several studies it was shown that single and multiple step surface derivatizations could be easily carried out on immobilized SPIONs. Apart from facilitated purification steps, all functionalizations were superior to liquid-phase synthesis in terms of particle recovery rate, size distribution, and reaction yield.

For any biological or medical application of such magnetic nanoparticles, the basic mechanism of their interaction with living matter needs to be investigated and understood. However, our understanding in this field has not kept pace with the extremely rapid development of new and highly complex nanoparticles. ${ }^{[17]}$ The reasons for this are many. For one, the system (i.e. ultrasmall particles in a biological environment) itself is extremely complex, with particles approaching the size of proteins, and the detailed investigation requires new models and possibly new analytical methods. In addition, highly multidisciplinary teams are required to approach this challenging task from different angles.

In the last decade, a plethora of studies have been performed in which the interaction of a large variety of different nanoparticles with cells or living organisms has been investigated. Since (nano)particles and cells, as well as the specific experimental conditions vary strongly between many of these studies, comparison is extremely challenging. We have seen in the past that nanoparticle size matters for nanoparticlecell interaction. Maybe even more importantly, surface matters. An important step in this area has been taken by Cederval $e t$ $a l .{ }^{[18,19]}$ on the development of methods for probing the association of proteins to nanoparticles. When particles enter a complex biological environment, it is hypothesized that proteins in this biological fluid quickly associate with the nanoparticles, which will have two effects. ${ }^{[20]}$ First, the surface of, for example, polymer-coated magnetic nanoparticles is modified by the adsorption of proteins and the interaction of those protein-coated NPSs with living cells, for example, might be different com-

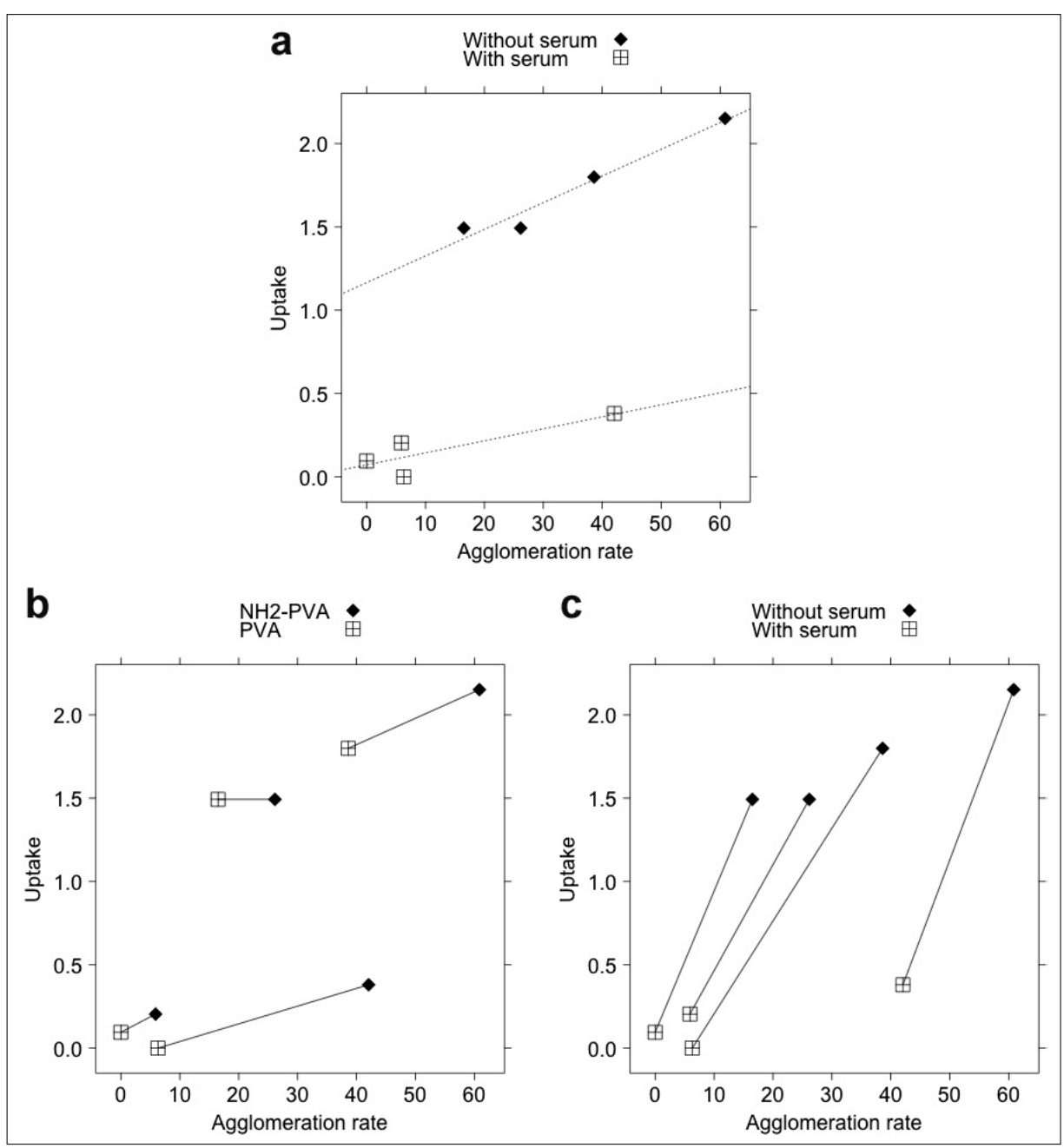

Fig. 8. Impact of colloidal stability (agglomeration rate) on cell uptake. a) A positive correlation of colloidal stability and cell uptake is observed for particles with and without serum. In general, increased aggregation leads to enhanced cell uptake. b) Influence of particle surface on stability and (indirectly) on cell uptake. Points connected by a line represent pairs of samples that were measured under the same experimental conditions, varying only in particle surface coating (PVA vs NH2-PVA). c) Influence of serum on stability and (indirectly) on cell uptake.

pared to the 'as-synthesized' nanoparticlecell interaction. Second, the surface coating can change the adsorbed protein, for example it can lead to altered conformation, perturbed function, or avidity effects arising from the close spatial repetition of the same protein. ${ }^{[17]}$ Investigating how proteins adsorb to positively, negatively and neutrally charged polymer coated SPOINs has shown only minor differences in the type and concentration of resultant NPprotein complexes due to the high affinity of abundant serum proteins, such as albumin, towards all NP surfaces, independent of their charge. However, adsorption rates varied significantly between the differently surface-charged particles. ${ }^{[21]}$ Despite such small differences between the specific proteins adsorbed, assessment of the subsequent cellular interaction has observed that positively surface-charged polymercoated SPIONs can be internalized rapidly and at a higher concentration compared to both the negatively and neutrally surfacecharged particles.[22-24] The same trend was observed using the same polymer coating on different core materials such as gold and polystyrene. ${ }^{[21]}$ In earlier studies we have shown that increasing the positive charge also had an important impact on cell viability. ${ }^{[25]}$ The correlation between particle surface, aggregation rate and cell uptake is illustrated in Fig. 8, showing a comparison of SPIONs coated with polyvinylalcohol (PVA) and SPIONs coated with vinyl alcohol-vinyl amine copolymer (NH2-PVA). Nanoparticle synthesis and characterization were performed as previously described. ${ }^{[11]}$ Both polymer-coated particles showed a diameter of around 30 $\mathrm{nm}$ (as obtained by dynamic light scattering) and a zeta potential of $+30 \mathrm{mV}(\mathrm{NH} 2-$ PVA) and $+1.5 \mathrm{mV}$ (PVA), respectively. The agglomeration behavior of both particle types was evaluated in cell culture medium in the presence and absence of fetal calf serum. The agglomeration rate $[\mathrm{nm} / \mathrm{h}]$ was determined as the slope between the measured particle sizes at two time points $(\mathrm{t}=0$ and $\mathrm{t}=2 \mathrm{~h})$. Both investigated 
SPIONs were long-term stable in standard buffers. In parallel, we studied the uptake by HeLa cells using the Prussian blue reaction $(120 \mu \mathrm{g} \mathrm{Fe} / \mathrm{ml})$. Fig. 8 summarizes the results of these experiments. Fig. 8a implies that there is a relation between agglomeration rate and uptake. In general, positively charged NH2-PVA-coated SPIONs aggregated faster than the neutral PVA-coated particles and hence showed also a higher uptake (Fig. 8b). The addition of serum (Fig. 8c) significantly increased the colloidal stability of the particles ( $\mathrm{p}=$ 0.02 ) while showing significantly lower cell uptake $(p=0.01)$. In conclusion, we observed that positively charged SPIONs elicited a lower colloidal stability (which in turn depended on the presence of serum proteins) than neutral polymer-coated SPIONs. It can be hypothesized that this characteristic is a causative factor within the NP cellular interaction, as it may promote a concentrated sedimentation, within an aggregated form, of NPs upon cells in vitro. This hypothesis is supported by another study, where we showed that particle uptake was significantly increased in the presence of a permanent magnet, which increased the local SPION concentration on the cell surface very rapidly.

Apart from correlating cell uptake to particle properties, many reports have addressed the issue of how nanoparticles may influence cellular responses (such as cytotoxicity, oxidative stress, inflammation and genotoxicity). ${ }^{[26]}$ Numerous studies have been published concerning the biocompatibility and possible adverse effects associated with SPIONS exposure both in vitro and in vivo. ${ }^{[27]}$ However, it is difficult to compare the plethora of publications due to their lack of NP characterization and different assays employed (i.e. biological system or endpoint test), as well as different (often too high) concentrations used, thus, it has been recognized that such studies need to fulfill standards such as proper characterization of the nanomaterial, the use of realistic concentrations and exposure scenarios, and a detailed description of the methods used. ${ }^{[28]}$ Another methodological challenge is the analysis of $\mathrm{NP}-$ cell interaction. This requires an interdisciplinary approach involving material characterization, cell biology (biochemistry) and microscopy. The first hurdle is to provide information regarding the NP size distribution, agglomeration state, shape, and surface charge, ${ }^{[29]}$ as these factors are essential to understanding the behavior of NPs at the biological interface. The second hurdle is to precisely detect and localize the intracellular NPs, as such knowledge provides imperative information pertinent to how NPs may enter cells, as well as their subsequent intracellular fate and cellular response. ${ }^{[30]}$ Due to their small size the
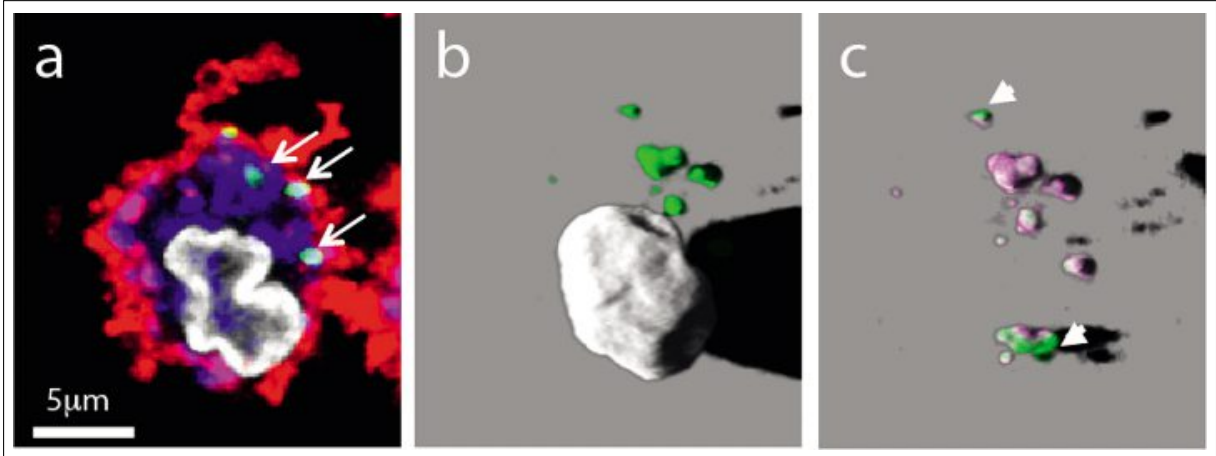

Fig. 9. Visualization of SPIONs using laser scanning microscopy. a) After exposure of dendritic cells to SPIONs for $4 \mathrm{~h}$ the cells have been fixed and labeled for laser scanning microscopy as described. A representative XY-section is shown with the F-Actin in red, cell nuclei in white, endosomal compartments in blue and the SPIONs in green. The F-actin staining facilitated detection of intracellular (arrows) localization of SPIONs. b) represents a 3D shadow reconstruction of the cell nuclei (white) and the SPIONs (green), whereas c) represents the cell nuclei (white) and the colocalized channel of SPIONs with endosomes (purple). Some of the SPIONs did not colocalize with endosomal compartments (arrowheads).

identification of NP within cells requires cutting-edge microscopy techniques. For fluorescently labeled NPs, laser scanning microscopy combined with digital image restoration (i.e. deconvolution algorithm) can be applied (Fig. 9). ${ }^{[31]}$ With such a technique it is possible to increase the resolution 2-3 times which is usually limited by light microscopy to $200 \mathrm{~nm}$ in the lateral, and 500-900 $\mathrm{nm}$ in the axial dimension. [32] The spatial and intracellular localization of such fluorescent NPs can also be analyzed more thoroughly, either in living or fixed cells, by performing co-localization studies with fluorescently labeled compartments such as endosomes, lysosomes, mitochondrion or the nucleus. ${ }^{[33]}$ The detection of electron-dense NPs is possible via transmission electron microscopy which has a resolution range from Angstrom to $\mathrm{nm}$. In addition to conventional transmission electron microscopy more sophisticated methods, such as energy filtered transmission electron microscopy which allows the identification of the chemical composition of a particle, [34] or electron tomography which enables the NPs to be visualized in three dimensions within cells should be applied. ${ }^{[35]}$ The third hurdle is quantification; understanding the spatial distribution of NPs within a defined reference volume (i.e. an intracellular compartment). ${ }^{[36]}$ Laser scanning microscopy offers a valuable tool to analyze the distribution of NPs within complete cells and their intracellular compartments. ${ }^{[37]}$

However, considering the resolution limit of light microscopy it is not possible to distinguish between single particles and small NP agglomerates. For transmission electron microscopy, stereological methods allow the assessment and quantification of NP distribution within cells. Although this method is challenging and time consuming, it offers a great tool to correlate functional data with NP intracellular localization. ${ }^{[38]}$

Since the SPIONs described above consist of an iron oxide core which is electron dense, and a hydrophilic polymer shell[11] to which a fluorescent dye can be coupled they can be visualized with different imaging techniques (i.e. light and electron microscopy). One of our research focuses is to understand the interaction of SPIONs with immune cells, for example dendritic cells (DCs), which are known to be one of the most immune competent cells within the human body. ${ }^{[39]}$ Following the exposure of SPIONs to dendritic cells, we have fixed and prepared the cells for transmission electron microscopy. The resultant images have shown that SPIONS can localize within vesicles of different sizes, which correspond to endosomal compartments. By applying laser scanning microscopy and co-localization analysis we could also show a dose-dependent uptake of the SPIONs by dendritic cells and that the majority of SPIOsS were localized inside endosomes, stained with the transferrin receptor. However, few NPs were detected inside the cytoplasm ${ }^{[40]}$ suggesting that additional endocytotic mechanisms may also play a key role in the cellular uptake of SPIONs. Further studies are necessary in order to quantify the intracellular trafficking. Interestingly, the antigen processing, and the capacity of DCs to stimulate CD4+ T-cells was decreased in the presence of SPIONS, revealing an immunemodulating (i.e. a suppressive effect). ${ }^{[40]}$ It is imperative that further research is performed regarding the concept of nanoimmunomodulation, since this field offers a completely new perspective for the use of SPIONs as an advantageous tool within nanomedicine. 


\section{Acknowledgements}

Part of the work outlined above was supported by the Swiss National Science Foundation (grants PP00P2_123373, 205321111908, 3200B0-104508, 406240_126104, CRSII2_130414，406440_131266 / 1, 320030_138365 / 1), by the European Union FP7 project NanoDiaRa, the German Research Foundation (SPP1313), Swiss Nanoscience Institute (SNI) within the National Center of Research (NCCR) in Nanoscale Science, the University of Fribourg, and the Adolphe Merkle Foundation. The results summarized in this article were obtained from numerous collaborations within the University of Fribourg, the University of Geneva, the Ecole Polytechnique Fédérale de Lausanne, and the University of Bern.

Received: January 31, 2012

[1] P. Rivera Gil, W. J. Parak, ACS Nano 2008, 2, 2200.

[2] 'The Nanotech Report', 4th ed. Lux Research, 2006, http://www.luxresearchinc.com/.

[3] Q. Pankhurst, J. Connolly, S. Jones, J. Phys. D: Appl. Phys. 2003, 36, 167.

[4] R. Hiergeist, W. Andra, N. Buske, R. Hergt, I. Hilger, U. Richter, W. Kaiser, J. Magnetism Magn. Mater. 1999, 201, 420.

[5] A. Jordan, K. Maier-Hauff, P. Wust, M. Johannsen, 'Nanomaterials for Cancer Therapy (Nanotechnologies for the Life Sciences)', Ed. C. S. S. R. Kumar, 1st ed. Wiley-VCH, Weinheim, 2006, p. 434

[6] A. Jordan, P. Wust, H. Fähling, W. John, A Hinz, R. Felix, Int. J. Hyperthermia 2009, 25, 499.

[7] P.-E. Le Renard, O. Jordan, A. Faes, A. PetriFink, H. Hofmann, D. Ruefenacht, F. Bosman, F. Buchegger, E. Doelker, Biomaterials 2010, 31,691 .
[8] P.-E. Le Renard, R. Lortz, C. Senatore, J.P. Rapin, F. Buchegger, A. Petri-Fink, H. Hofmann, E. Doelker, O. Jordan, J. Magnetism Magn. Mater. 2011, 323, 1054.

[9] P.-E. Le Renard, F. Buchegger, A. Petri-Fink, F. Bosman, D. Rüfenacht, H. Hofmann, E. Doelker, O. Jordan, Int. J. Hypothermia 2009. 25, 229.

[10] A.-H. Lu, E. L. Salabas, F. Schüth, Angew. Chem. Int. Ed. 2007, 46, 1222.

[11] M. Chastellain, A. Petri, H. Hofmann, J. Colloid Interface Sci. 2004, 278, 353.

[12] S. Sun, H. Zeng, J. Am. Chem. Soc. 2002, 124, 8204.

[13] R. Digigow, H. Dietsch, B. Rothen-Rutishauser, A. Fink-Petri, in preparation.

[14] R. Kodama, J. Magnetism Magn. Mater. 1999. $200,359$.

[15] S. McNeil, J. Leukocyte Bio. 2005, 78, 585.

[16] B. Steitz, J. Salaklang, A. Finka, C. O’Neil, H. Hofmann, A. Petri-Fink, Bioconjugate Chem. 2007, 18, 1684

[17] J. P. Klein, Nat. Acad. Sci. USA 2007, 104 2029.

[18] T. Cedervall, I. Lynch, S. Lindman, T. Berggård, E. Thulin, H. Nilsson, K. A. Dawson, S. P. Linse, Nat. Acad. Sci. USA 2007, 104, 2050.

[19] P. Aggarwal, J. B. Hall, C. B. McLeland, M. A. Dobrovolskaia, S. E. McNeil, Adv. Drug Delivery Rev. 2009, 61, 428 .

[20] I. Lynch, T. Cedervall, M. Lundqvist, C. Cabaleiro-Lago, S. Linse, K. A. Dawson, $A d v$. Colloid Interfac. 2007, 134-135, 167.

[21] V. Hirsch, M. Clift, B. Rothen-Rutishauser, in preparation.

[22] A. Petri-Fink, M. Chastellain, L. JuilleratJeanneret, A. Ferrari, H. Hofmann, Biomater. 2005, 26, 2685

[23] F. Cengelli, D. Maysinger, F. Tschudi-Monnet, X. Montet, C. Corot, A. Petri-Fink, H. Hofmann, L. Juillerat-Jeanneret, J. Pharmacol. Exp. Ther. 2006, 318, 108 .

[24] A. Petri-Fink, B. Steitz, A. Finka, J Salaklang, H. Hofmann, Eur. J. Pharmaceut. Biopharmaceut. 2008, 68, 129.
[25] A. Petri-Fink, H. Hofmann, IEEE Trans. Nanobiosci. 2007, 6, 289.

[26] K. Donaldson, L. Tran, L. A. Jimenez, R. Duffin, D. E. Newby, N. Mills, W. MacNee, V. Stone, Part. Fibre Toxicol. 2005, 2, 10.

[27] M. Mahmoudi, H. Hofmann, B. RothenRutishauser, A. Petri-Fink, Chem. Rev. 2011, 111230103921008.

[28] H. F. Krug, P. Wick, Angew. Chem. Int. Ed. 2011, 50, 1260.

[29] G. Oberdörster, E. Oberdörster, J. Oberdörster, Environ. Health Perspect. 2005, 113, 823

[30] C. Muehlfeld, B. Rothen-Rutishauser, F. Blank, D. Vanhecke, M. Ochs, P. Gehr, Am. J. Physiol. Lung C 2008, 294, L817.

[31] M. J. D. Clift, J. Varet, S. M. Hankin, B. Brownlee, A. M. Davidson, C. Brandenberger, B. Rothen-Rutishauser, D. M. Brown, V. Stone, Nanotoxicol. 2011, 5, 664

[32] H. T. M. Voort, K. C. Strasters, J. Microsc. 2011, $178,165$.

[33] A. Lehmann, W. Parak, F. Zhang, Z. Ali, C. Röcker, Small 2010, 6, 753.

[34] C. Brandenberger, M. J. D. Clift, D. Vanhecke, C. Muehlfeld, V. Stone, P. Gehr, B. RothenRutishauser, Part. Fibre Toxicol. 2010, 7, 15.

[35] M. J. D. Clift, E. J. Foster, D. Vanhecke, D Studer, P. Wick, P. Gehr, B. Rothen-Rutishauser, C. Weder, Biomacromol. 2011, 12, 3666.

[36] C. Mühlfeld, T. M. Mayhew, P. Gehr, B. RothenRutishauser, J. Aerosol Med. 2007, 20, 395.

[37] B. Rothen-Rutishauser, F. Blank, A. Fink-Petri, M. J. D. Clift, T. Geiser, C. von Garnier, G.I.T Imaging \& Microscopy 2011, March 29, p. 30.

[38] C. Brandenberger, C. Muehlfeld, Z. Ali, A.G. Lenz, O. Schmid, W. J. Parak, P. Gehr, B. Rothen-Rutishauser, Small 2010, 6, 1669.

[39] L. Nicod, Eur. Respiratory Rev. 2005, 14, 45.

[40] F. Blank, P. Gerber, B. Rothen-Rutishauser, U. Sakulkhu, J. Salaklang, K. De Peyer, P. Gehr, L. P. Nicod, H. Hofmann, T. Geiser, A. Petri-Fink, C von Garnier, Nanotoxicol. 2011, 5, 606. 